TRANSACTIONS OF THE

AMERICAN MATHEMATICAL SOCIETY

Volume 223, 1976

\title{
COMPACT NILMANIFOLD EXTENSIONS OF ERGODIC ACTIONS
}

\author{
BY
}

ROBERT J. ZIMMER

\begin{abstract}
We study extensions of dymanical systems defined by cocycles into nilpotent Lie groups.
\end{abstract}

1. Introduction. An important class of flows in ergodic theory are those defined by one-parameter subgroups acting on a homogeneous space of a locally compact group. If the homogeneous space is actually a compact abelian group, the resulting flows are a model for those with discrete spectrum and are reasonably well understood. The case in which the homogeneous space is a compact nilmanifold was studied in detail in [1], and studies have subsequently been made for the case of homogeneous spaces of solvable and semisimple Lie groups. (See [2] and [8], for example.) It was pointed out by Mackey [7] that a natural generalization of this question was to begin with a fixed ergodic flow and to consider cocycles of the flow taking values in various locally compact groups. In this way, one obtains "homogeneous space extensions" of the original flow, in which the fibers are a fixed homogeneous space. The case in which the homogeneous space is a quotient space of a compact group is of central importance in the notions of relatively discrete spectrum and generalized discrete spectrum, discussed by the author in [10], [11]. In this paper, we begin consideration of the case in which the homogeneous space is a compact nilmanifold. Two important questions about nilflows considered in [1] are the (related) questions of ergodicity and identification of the discrete spectrum. The point of this note is to obtain analogous results concerning the questions of ergodicity of extensions and identification of the relative discrete spectrum.

As is often the case in the study of extensions, the reader will gain by examining the results from the virtual subgroup viewpoint [7], although no special knowledge of this notion is required for reading this paper. $\$ 2$ below contains preliminary material, concerning the space of finite dimensional subspaces of a Hilbert space, which will be used in the main part of the paper, \$3. \$3 uses many of the notions of [10], [11], and the reader is referred there for undefined terms.

Received by the editors October 24, 1975.

AMS (MOS) subject classifications (1970). Primary 22E25, 28 A65. 
2. The Borel space of finite dimensional subspaces. In this section, we examine properties of the space of finite dimensional subspaces of a separable Hilbert space $H$, with dimension less than a fixed integer. In particular, we will show that it can be given the structure of a complete separable metric space, and that the natural action of $U(H)$, the unitary group of $H$, on this space is jointly continuous, if $U(H)$ has the strong operator topology. Finally, we will show that for certain subgroups of $U(H)$, the quotient space under the natural action of this subgroup is a standard Borel space. It is this type of result that will prove useful in the study of ergodic extensions.

Let $B$ be the closed unit ball in $H$. Then $B$ is a bounded complete separable metric space, with the metric $d$ induced by the norm. Let $C$ be the set of closed nonempty subsets of $B$. Various notions of convergence in $C$ have been investigated. $C$ is a complete metric space with the Hausdorff metric $\rho$ defined by

$$
\rho(E, F)=\max \left\{\sup _{x \in E} d(x, F), \sup _{y \in F} d(y, E)\right\} .
$$

(See [6, §§15.VII and 29.IV].) Another notion of convergence, which has been investigated by Effros [3], can be described as follows: Let $F_{n}$ be a sequence in C. Define

$$
\lim \inf F_{n}=\left\{x \in B \mid x=\lim x_{n}, \text { for some } x_{n} \in F_{n}\right\}
$$

and

$$
\lim \sup F_{n}=\left\{x \in B \mid x=\lim x_{n_{j}},\right.
$$

$$
\text { where } x_{n_{j}} \in F_{n_{j}} \text { for some subsequence } F_{n_{j}} \text { of } F_{n} \text { \}. }
$$

Finally, define $F_{n} \rightarrow F$ if $F=\lim \sup F_{n}=\lim \inf F_{n}$. As pointed out by Effros $\left[3\right.$, p. 930] it is easy to check that $\rho\left(F_{n}, F\right) \rightarrow 0$ implies $F_{n} \rightarrow F$.

Now let $F^{k}$ be the set of subspaces of $H$ of dimension $\leqslant k$ and $F_{k}=$ $\left\{V \in C \mid V=B \cap V^{\prime}\right.$ for some $\left.V^{\prime} \in F^{k}\right\}$.

Proposition 2.1. $F_{k}$ is closed in $(C, \rho)$.

Proof. If $V_{n} \in F_{k}$ and $V_{n} \rightarrow A, A \in C$, it is straightforward that $A$ is of the form $A^{\prime} \cap B$, where $A^{\prime} \subset H$ is a subspace. To see that $\operatorname{dim} A^{\prime} \leqslant k$, suppose there are $k+1$ orthonormal elements in $A$, say $v_{1}, \ldots, v_{k+1}$. For each $i, v_{i}=$ $\lim _{n} v_{i n}$, where $v_{i n} \in V_{n}$. Thus, given any $\epsilon$, we can find $n$ with $\left\langle v_{i n} \mid v_{p n}\right\rangle<\epsilon$ for all $p \neq i, 1 \leqslant i, p \leqslant k+1$, and $\left\langle v_{i n} \mid v_{i n}\right\rangle>1-\epsilon$. Since $\operatorname{dim} V_{n} \leqslant k$ for all $n$, this is clearly impossible.

Proposition 2.2. $F_{k}$ is separable.

Proof. Let $S$ be a countable dense set of the boundary of $B$, and let $T=$ $\{A \subset S \mid \operatorname{card}(A) \leqslant k\}$. Then $T$ is also countable. For each $A \in T$, let $[A]=$ $\operatorname{span}(A) \cap B$, so that $[T]$ is a countable subset of $F_{k}$. If $V \in F_{k}$, let 
$v_{1}, \ldots, v_{n}, n \leqslant k$, be an orthonormal basis of $\operatorname{span}(V)$. For each $j$, choose $s_{i j} \in S$ such that $d\left(v_{i}, s_{i j}\right)<j^{-1}$. One can readily verify, using standard techniques in the geometry of Hilbert space, that $\rho\left(\left[s_{i j}, \ldots, s_{n j}\right], V\right) \rightarrow 0$ as $j \rightarrow \infty$. Thus $T$ is dense in $F_{k}$.

Corollary 2.3. $F_{k}$ is a complete separable metric space with the Hausdorff metric.

$F_{k}$ thus becomes a standard Borel space, with the Borel structure induced by the topology. It will be important for us to know when a map from a given standard Borel space into $F_{k}$ is Borel. The following result, though not precisely in this form, is essentially due to Effros.

Proposition 2.4. Let $S$ be a standard Borel space and suppose $v_{i}: S \rightarrow B$, $i=1, \ldots, p, p \leqslant k$, such that $\left\{v_{i}(s)\right\}_{i=1, \ldots, p}$ is orthonormal for each $s$. Then $s \rightarrow F_{k}$ defined by $s \rightarrow \operatorname{span}\left(\left\{v_{i}(s)\right\}\right) \cap B$ is Borel.

Proof. $F^{k}$ and $F_{k}$ each have a topology defined by the convergence closed subsets [3, p. 929] and it is easy to see that the map $r: F^{k} \rightarrow F_{k}, r(X)=$ $X \cap B$, is a homeomorphism. Thus $r$ is a Borel isomorphism for the corresponding Borel structures, which we call the convergence Borel structures. Now the proof of the theorem of $[3, \mathrm{pp} .930,931]$, shows that the convergence Borel structure on $F_{k}$ is the same as the Borel structure induced by the Hausdorff metric. Thus, to show $s \rightarrow \operatorname{span}\left(\left\{v_{i}(s)\right\}\right) \cap B$ is Borel, it suffices to show that $s \rightarrow F^{k}, s \rightarrow \operatorname{span}\left(\left\{v_{i}(s)\right\}\right)$ is Borel, where $F^{k}$ has the convergence Borel structure. The corollary to [4, Theorem 2] shows that this is Borel if there are countably many Borel maps $w_{j}: S \rightarrow H$ such that $\left\{w_{j}(s)\right\}_{j}$ is dense in $\left(\operatorname{span}\left\{v_{i}(s)\right\}\right)^{\perp}$. But for each $w \in H, w(s)=w-\Sigma\left\langle w \mid v_{i}(s)\right\rangle v_{i}(s)$ is Borel and lies in $\left(\operatorname{span}\left\{v_{i}(s)\right\}\right)^{\perp}$. Choosing a countable dense set of $w$ 's in $H$ produces the required functions.

We now examine the natural action of the unitary group on $F_{k}$.

Proposition 2.5. Let $U(H)$ be the unitary group of $H$. Then the left action of $U(H)$ on $F_{k}$ defined by $U(H) \times F_{k} \rightarrow F_{k},(U, V) \rightarrow U(V)$ is jointly continuous, where $U(H)$ has the strong operator topology.

Proof. Suppose $U_{n} \rightarrow U$ and $\rho\left(V_{n}, V\right) \rightarrow 0$. We have $\rho\left(U_{n}\left(V_{n}\right), U(V) \leqslant\right.$ $\rho\left(U_{n}\left(V_{n}\right), U_{n}(V)\right)+\rho\left(U_{n}(V), U(V)\right)$. It is straightforward to check that $\rho\left(U_{n}\left(V_{n}\right), U_{n}(V)\right)=\rho\left(V_{n}, V\right) \rightarrow 0$. Hence, it suffices to show that $\rho\left(U_{n}(V), U(V)\right) \rightarrow 0$. Let $v_{1}, \ldots, v_{p}$ be an orthonormal basis of $\operatorname{span}(V)$, and let $\epsilon>0$. Then for $n$ sufficiently large, we have $d\left(U_{n} v_{i}, U v_{i}\right)<\epsilon / \sqrt{p}$ for each $i=1, \ldots, p$. Let $x \in U(V)$, so $x=\Sigma a_{i} U v_{i}$. Let $x_{n} \in U_{n}(V)$ be $x_{n}=$ $\Sigma a_{i} U_{n} v_{i}$. Then 


$$
\begin{aligned}
\left\|x-x_{n}\right\| & =\left\|\sum_{i} a_{i}\left(U v_{i}-U_{n} v_{i}\right)\right\| \\
& \leqslant\left(\sum\left|a_{i}\right|^{2}\right)^{1 / 2}\left(\sum\left\|U v_{i}-U_{n} v_{i}\right\|^{2}\right)^{1 / 2} \\
& \leqslant 1 \cdot\left(p \epsilon^{2} / \rho\right)^{1 / 2}=\epsilon .
\end{aligned}
$$

Hence, for $n$ sufficiently large, $d\left(x, U_{n}(V)\right) \leqslant \epsilon$ for all $x \in U(V)$, and the same argument shows that $d(y, U(V)) \leqslant \epsilon$ for all $y \in U_{n}(V)$ ( $n$ sufficiently large). Hence $\rho\left(U(V), U_{n}(V)\right) \rightarrow 0$, completing the proof.

We now specialize to the case where $H=L^{2}\left(\mathrm{R}^{n}\right)$. Let $U_{t}\left(t \in \mathrm{R}^{n}\right)$ denote the regular representation of $\mathbf{R}^{n}$ on $H$.

LEMMA 2.6. Let $g \in L^{2}\left(\mathbf{R}^{n}\right),\|g\|=1$, and $V \subset L^{2}\left(\mathbf{R}^{n}\right)$ be a finite dimensional subspace. Given any $\epsilon>0$, there is an $N$ such that $\|t\| \geqslant N$ implies $\left\|U_{t} f-g\right\|>1-\epsilon$, for all $f \in V$ with $\|f\|=1$.

Proof. Let $f_{1}, \ldots, f_{p}$ be an orthonormal basis of $V$. We can choose $R>0$ so that for $A=\{x \mid\|x\|>R\}, \int_{A}\left|f_{i} \bar{f}_{j}\right|<\epsilon / 2 p^{2}$ for all $i, j$ and $r>0$ so that, for $D=\{x \mid\|x\|>r\}, \int_{D}|g|^{2}<\epsilon / 2$. Suppose $f \in V$ and $\|f\|=1$. Write $f=\Sigma a_{i} f_{i}$, with $\left|a_{i}\right| \leqslant 1$. Then

$$
\begin{aligned}
\int_{A}|f|^{2} & =\int_{A}\left|\sum a_{i} f_{i}\right|^{2}=\int_{A}\left|\sum a_{i, j} \bar{a}_{j} f_{i} \bar{f}_{j}\right| \\
& \leqslant \sum \int_{A}\left|f_{i} \bar{f}_{j}\right| \leqslant p^{2} \frac{\epsilon}{2 p^{2}}=\frac{\epsilon}{2} .
\end{aligned}
$$

Now choose $N>R+r$, and let $t \in \mathrm{R}^{n}$ with $\|t\|>N$. Then $\mathrm{R}^{n}-D \subset A-t$. Since $\left\|U_{t} f-g\right\|^{2} \geqslant 2-2\left|\left\langle U_{t} f \mid g\right\rangle\right|$, it suffices to show that $\left|\left\langle U_{t} f \mid g\right\rangle\right| \leqslant \epsilon$. But

$$
\begin{aligned}
\left|\int U_{t} f \cdot \bar{g}\right| \leqslant & \int_{D}\left|U_{t} f\right||g|+\int_{\mathrm{R}^{n}-D}\left|U_{t} f\right||g| \\
\leqslant & \left(\int_{D}\left|U_{t} f\right|^{2}\right)^{1 / 2}\left(\int_{D}|g|^{2}\right)^{1 / 2} \\
& +\left(\int_{A-t}\left|U_{t} f\right|^{2}\right)^{1 / 2}\left(\int_{A-t}|g|^{2}\right)^{1 / 2} \\
\leqslant & \left\|U_{t} f\right\| \frac{\epsilon}{2}+\frac{\epsilon}{2}\|g\| \leqslant \epsilon .
\end{aligned}
$$

Proposition 2.7. Let $\mathbf{R}^{n}$ act on $F_{k}$ by $\mathbf{R}^{n} \times F_{k} \rightarrow F_{k},(t, V) \rightarrow U_{t}(V)$. Then the action is jointly continuous and the orbits are locally closed.

Proof. Continuity follows from Proposition 2.4 and the strong continuity of the regular representation. To see that the orbits are locally closed, let $V \in$ $F_{k}$. If $V=\{0\}$, the orbit of $V$ is clearly closed. So suppose $V \neq\{0\}$, and let $\Omega$ be the open set $F_{k}-\{0\}$. We claim orbit $(V) \cap \Omega$ is closed in $\Omega$, which suffices to conclude the result. So suppose there is a sequence $t_{j} \in \mathbf{R}^{n}$ such that $\rho\left(U_{t_{j}}(V), W\right) \rightarrow 0$ as $j \rightarrow \infty$, for some $W \in \Omega$. Choose $x \in W,\|x\|=1$. Then 
there is a sequence $v_{j} \in V$ with $U_{t_{j}}\left(v_{j}\right) \rightarrow x$ in norm. Dividing $v_{j}$ by $\left\|v_{j}\right\|$ if necessary, we can assume $\left\|v_{j}\right\|=1$. Suppose $\left\{t_{j}\right\}$ is unbounded. Then, by Lemma 2.6, for some subsequence $t_{j(k)}$, we will have $\left\|U_{t_{j(k)}}\left(v_{j(k)}\right)-x\right\| \geqslant 1 / 2$, which is impossible. Thus $\left\{t_{j}\right\}$ is bounded, and hence has a subsequence converging to some $t$. But then $W=U_{t}(V) \in \operatorname{orbit}(V)$.

COROLlARY 2.8. Let $\hat{F}_{k}$ be the space of orbits in $F_{k}$ under the action of $\mathbf{R}^{n}$. Then $\hat{F}_{k}$ is a standard Borel space with the quotient Borel structure.

Proof. This follows immediately from [5, Theorem 2.9].

3. Cocycles into nilpotent groups. We now turn to the subject proper of this paper, compact nilmanifold extensions.

We review some basic facts concerning extensions, cocycles, and cocycle representations [7], [10]. Let $G$ be a locally compact (second countable) group and let $S$ be an ergodic Lebesgue $G$-space. Thus, there is a Borel right $G$-action on $S$ and a probability measure $\mu$ on $S$ invariant and ergodic under $G$. Suppose $M$ is a standard Borel group. Then a function $\alpha: S \times G \rightarrow M$ is called a cocycle if for all $g, h \in G, \alpha(s, g h)=\alpha(s, g) \alpha(s g, h)$ for almost all $s \in S$. If $M=U(H)$, then $\alpha$ is called a cocycle representation of $S \times G$. Two cocycles $\alpha, \beta: S \times G$ $\rightarrow M$ are called equivalent or cohomologous if there is a Borel function $\phi: S \rightarrow$ $M$ such that, for each $g \in G, \phi(s) \alpha(s, g) \phi(s g)^{-1}=\beta(s, g)$ a.e. As pointed out by Mackey, the collection of (equivalence classes of) cocycle representations of a fixed $G$-space has a similar structure to the (equivalence classes of) unitary representations of a group. For example, if $\alpha: S \times G \rightarrow U\left(H_{1}\right)$ and $\beta: S \times G \rightarrow$ $U\left(H_{2}\right), \alpha \oplus \beta: S \times G \rightarrow U\left(H_{1} \oplus H_{2}\right)$ is defined by $(\alpha \oplus \beta)(s, g)=\alpha(s, g) \oplus$ $\beta(s, g)$. Infinite direct sums and tensor products can be similarly defined. We say that $\alpha$ is a subcocycle representation of $\gamma$ if $\gamma$ is equivalent to $\alpha \oplus \beta$ for some $\beta$. If $\gamma$ has no subcocycle representations $\gamma$ is called irreducible. On the other hand, if $\gamma=\Sigma^{\bullet} \gamma_{i}$ where $\gamma_{i}$ are finite dimensional, we say that $\gamma$ has discrete spectrum. Another, perhaps more geometric, way of looking at cocycle representations is as follows. If $\gamma: S \times G \rightarrow U(H)$, we let $H_{1}=\int_{S}^{\bullet} H d s=L^{2}(S ; H)$. Then $\gamma$ induces a unitary representation $U^{\gamma}$ of $G$ on $H_{1}$, defined by $\left(U^{\gamma} f\right)(s)=$ $\gamma(s, g) f(s g)$. This is simply translation by the $G$-action combined with a "twist" defined by $\boldsymbol{\gamma}$. Then subcocycle representations are in one-one correspondence with $U^{\boldsymbol{\gamma}}$-invariant fields of subspaces in $\int^{\oplus} H d s$.

Cocycle representations arise naturally in the study of extensions of ergodic actions. Namely, suppose $\phi:(X, \mu) \rightarrow(Y, \nu)$ is a measure preserving Borel map of $G$-spaces where $Y$ is ergodic. Decomposing $\mu$ with respect to $\nu$ over the fibers of $\phi, L^{2}(X)$ becomes a Hilbert bundle over $Y$, and there corresponds a naturally defined $Y \times G$ cocycle representation $[10, \S 2]$. One then hopes to study the extension $X \rightarrow Y$ by means of this cocycle. In particular, [10] and [11] were 
devoted to studying the situation in which the cocycle has subcocycle representations with discrete spectrum. The direct sum of the finite dimensional subcocycles is called the relatively discrete spectral part of $X$ over $Y$, and if this is all of $L^{2}(X)$, we say that $X$ has relatively discrete spectrum over $Y$.

Examples of extensions arise from cocycles into locally compact groups. Thus, if $\alpha: S \times G \rightarrow H$ is a cocycle with $H$ locally compact, and $H_{0} \subset H$ is a closed subgroup, then $\alpha$ defines an action of $G$ on $S \times H / H_{0}$ given (almost everywhere; see [10, Proposition $3.2 \mathrm{ff}$.]) by $(s,[h]) g=(s g,[h] \alpha(s, g))$. In this case, $L^{2}\left(S \times H / H_{0}\right)=\int_{S}^{\oplus} L^{2}\left(H / H_{0}\right) d s$ and the natural $S \times G$ cocycle representation of this extension is just $\pi \circ \alpha: S \times G \rightarrow U\left(L^{2}\left(H / H_{0}\right)\right)$ where $\pi$ is the natural representation of $H$ on $L^{2}\left(H / H_{0}\right)$, i.e. the representation induced by the one dimensional identity representation of $H_{0}$. If $H$ is compact, then $S \times H / H_{0}$ has relatively discrete spectrum over $S$. However, for noncompact $H$, this is no longer true in general.

In this paper, we consider the case in which $H=N$, a connected, simply connected nilpotent Lie group, and $D \subset N$ is a uniform discrete subgroup. We suppose that $\alpha: S \times G \rightarrow N$ is a cocycle, so that $S \times N / D$ is a $G$-space extension of $S$.

The two questions we shall consider here are (i) to obtain criteria for the ergodicity of $S \times N / D$, and (ii) to identify the relatively discrete spectrum of $S \times N / D$ as an extension of $S[10$, Definition 5.1]. Our approach is to apply results on the irreducible representations of nilpotent Lie groups to cocycles into these groups in a fashion similar to the way in which these results were applied to one-parameter subgroups in [1, Chapter V].

When $S$ is one point and $G=\mathbf{R}$, the cocycle defines a nilflow, and [1, Theorem V.4.2] shows that the nilflow on $N / D$ is ergodic if and only if the factor flow on $N / D[N, N]$ is ergodic, and that the subspace of $L^{2}(N / D)$ generated by the eigenfunctions of the flow is precisely $L^{2}(N / D[N, N])$. Corollaries 3.4 and 3.5 below are the natural generalization to extensions of these results. The reader is referred to [1] for unexplained results and notation about nilmanifolds.

We begin with a result we shall need about cocycles into $\mathbf{R}^{n}$.

Proposition 3.1. Suppose $\alpha: S \times G \rightarrow \mathrm{R}^{n}$ is a cocycle and $\gamma=U \circ \alpha$ where $U$ is the regular representation of $\mathbf{R}^{n}$. Then if $\gamma$ has a subcocycle with discrete spectrum $[10, \S 4]$, then $\alpha$ is equivalent to the zero cocycle.

Proof. If $\gamma$ has a subcocycle with discrete spectrum, then there is a $G$-invariant sub-Hilbert bundle, $s \rightarrow V(s)$, of the product bundle $s \rightarrow L^{2}\left(R^{n}\right)$, where $V(s)$ is finite dimensional for all $s$. By virtue of ergodicity, $\operatorname{dim} V(s)$ is constant on a conull Borel set, so changing $V(s)$ on a null Borel set, if necessary, we can assume $\operatorname{dim} V(s)=k$, for some $k<\infty$. By Proposition 2.4, $V: S \rightarrow F_{k}$ is Borel. 
Furthermore, $G$-invariance of $\{V(s)\}$ implies that, for each $g, \gamma(s, g)(V(s g))=$ $V(s)$ for almost all $s$. This can be written

$$
\alpha(s, g) \cdot V(s g)=V(s),
$$

where the left side denotes the action defined in Proposition 2.7. Let $p: F_{k} \rightarrow$ $\hat{F}_{k}$ be the quotient map onto the space of orbits. Then $p \circ V$ is Borel, and it follows from (*) that, for each $g,(p \circ V)(s)=(p \circ V)(s g)$ for almost all s. By Corollary $2.8, \hat{F}_{k}$ is standard, and it follows from the ergodicity of $G$ on $S$ that $p \circ V$ is constant on a conull Borel set $S_{0} \subset S$. Thus, there is some $\mathbf{R}^{n}$-orbit $A \subset F_{k}$ with $V(s) \in A$ for all $s \in S_{0}$. Changing $V$ on $S-S_{0}$, we can assume $V(s) \in A$ for all s. Now $\mathrm{R}^{n}$ acts transitively on $A$, so we can choose a Borel function $\lambda: A \rightarrow \mathrm{R}^{n}$, such that, for all $a \in A, \lambda(a) \cdot a=a_{0}$, for some fixed $a_{0} \in A$. Let $c: S \rightarrow \mathrm{R}^{n}$ be $c=\lambda \circ V$. Then $c$ is Borel and $c(s) \cdot V(s)=a_{0}$ for all $s \in S$. Define $\beta(s, g)=c(s)+\alpha(s, g)-c(s g)$. Then $\beta$ is a cocycle equivalent to $\alpha$, and we claim that, for each $g, U(\beta(s, g))\left(a_{0}\right)=a_{0}$ for almost all s. The left side is $[c(s)+\alpha(s, g)-c(s g)] \cdot a_{0}=[c(s)+\alpha(s, g)] \cdot V(s g)=c(s) \cdot V(s)$ $=a_{0}$. However, $a_{0}$ is a finite dimensional subspace of $L^{2}\left(R^{n}\right)$, and the only element of $\mathbf{R}^{n}$ that leaves a finite dimensional subspace invariant is 0 . (This follows from Lemma 2.6, for example.) So, for each $g, \beta(s, g)=0$ for almost all $s$, and this suffices to conclude the result.

Now let $\alpha: S \times G \rightarrow N$ be a cocycle and let $\sigma$ be the representation of $N$ induced by the one dimensional identity representation of $D$. Then $\sigma$ is the direct sum of irreducible representations of $N$, which are either one dimensional or infinite dimensional. Our main results will follow from

THEOREM 3.2. Let $\pi$ be an infinite dimensional irreducible subrepresentation of $\sigma$, acting in a Hilbert space $H$. Then if the cocycle representation $\pi \circ \alpha$ : $S \times G \rightarrow U(H)$ has a finite dimensional subcocycle representation, the action of $G$ on $S \times N / D[N, N]$ is not ergodic.

We first prove a lemma.

LEMMA 3.3. Let $A, B$ be locally compact groups and $h: A \rightarrow B$ a surjective homomorphism. Suppose $\alpha: S \times G \rightarrow A$ is a cocycle and that $h \circ \alpha$ is equivalent to the cocycle into the identity. Then $\alpha$ is equivalent to a cocycle into $\operatorname{ker}(h)$.

Proof. Let $\lambda: B \rightarrow A$ be a Borel section of $h$. Let $b: S \rightarrow B$ be a Borel map such that, for each $g, b(s)(h \circ \alpha)(s, g) b(s g)^{-1}=e$ for almost all s. Let $c=\lambda \circ b$. Then for each $g$ we have $c(s) \alpha(s, g) \subseteq(s g)^{-1} \in \operatorname{ker}(h)$ for almost all $s$.

Proof of Theorem 3.2. There is a subgroup $N_{0}$ of $N$ with $[N, N] \subset$ $N_{0} \subset N$ such that $N / N_{0} \cong \mathrm{R}^{n}$, and $\pi$ is induced by a one dimensional representa. 
tion $\chi$ of $N_{0}$ [1, Corollary V.3.2]. Furthermore, we can assume that the scale is chosen in $\mathrm{R}^{n}$ such that the quotient map $q: N \rightarrow \mathrm{R}^{n} \cong N / N_{0}$ satisfies $q_{n}(D) \neq 0$, $q_{n}(D) \subset Z$, the group of integers, where $q_{n}$ is the $n$th coordinate function of $q$. Let $\beta: \mathbf{R}^{n} \times N \rightarrow T^{1}$ (the unit circle) be a cocycle corresponding to the representation $\chi$ of $N_{0}[9$, Theorem 8.27]. Then we can realize the cocycle $\rho=\pi \circ \alpha$ as follows: $H=L^{2}\left(\mathbf{R}^{n}\right)$, and for each $f \in H, t \in \mathbf{R}^{n}$,

$$
(\rho(y, g) f)(t)=\beta(t, \alpha(y, g)) f(t+q(\alpha(y, s))) .
$$

Now let $U$ be the regular representation of $\mathbf{R}^{n}$ and define the cocycle $\gamma=U \circ q \circ \alpha$; i.e. for $f \in L^{2}\left(\mathbf{R}^{n}\right),(\gamma(y, g) f)(t)=f(t+q(\alpha(y, g)))$. Since $\beta \bar{\beta}=1$, it follows in a straightforward manner that $\rho \otimes \bar{\rho}=\gamma \otimes \bar{\gamma}$ on $L^{2}\left(R^{n}\right)$ $\otimes L^{2}\left(\mathbf{R}^{n}\right) \cong L^{2}\left(\mathbf{R}^{n} \times \mathrm{R}^{n}\right)$. It follows from [10, Lemma 2.13] that $\rho$ has a finite dimensional subcocycle representation if and only if $\boldsymbol{\gamma}$ does. However $q \circ \alpha: S \times G \rightarrow \mathbf{R}^{n}$ is a cocycle, and Proposition 3.1 implies that $q \circ \alpha$ must be equivalent to the zero cocycle. From Lemma 3.3, we then conclude that $\alpha$ must be equivalent to a cocycle $c$ taking values in $K=\operatorname{ker}(q)$. To show that the action on $S \times N / D[N, N]$ defined by $\alpha$ is not ergodic, it suffices to show that the action defined by $c$ is not ergodic [10, Corollary 3.4] $D[N, N]$ is a normal subgroup of $N$ and we let $\phi: N \rightarrow N / D[N, N]$ be the quotient homomorphism. To show that the action defined by $c$ is not ergodic, it suffices to show that $(\phi \circ c)(S \times G)$ is contained in a proper closed subgroup of $N / D[N, N][10$, Corollary 3.8$]$.

To this end, we let $K_{0}=\operatorname{ker}\left(q_{n}\right)$; we claim $D K_{0}$ is closed. So suppose $d_{j} \in D, x_{j} \in K_{0}$ and $d_{j} x_{j} \rightarrow x \in N$. Then $q_{n}(D) \subset Z$ and $q_{n}\left(K_{0}\right)=0$, so $q_{n}\left(d_{j}\right)=q_{n}\left(d_{j} x_{j}\right) \rightarrow q_{n}(x)$. Thus $q_{n}\left(d_{j}\right)$ is eventually constant, so we can in fact assume it is constant. Let $e_{j} \in N$ with $d_{j}=d_{1} e_{j}$. Then $e_{j} \in K_{0}$ and $d_{1} e_{j} x_{j}$ $\rightarrow x$. Thus $e_{j} x_{j} \rightarrow d_{1}^{-1} x$, and since $K_{0}$ is closed, $d_{1}^{-1} x \in K_{0}$. Hence $x \in D K_{0}$, showing the latter is closed. Since $K_{0} \supset[N, N], D K_{0} \supset D[N, N]$, it follows that $\phi\left(D K_{0}\right)$ is a closed subgroup of $N / D[N, N]$. Since $D K_{0} \neq N$ (otherwise $q_{n}(N) \subset Z$, which is impossible by the connectedness of $\left.N\right), \phi\left(D K_{0}\right) \neq N / D[N, N$. Lastly, it is clear that $(\phi \circ c)(S \times G) \subset \phi\left(D K_{0}\right)$. This completes the proof.

COROLlaRY 3.4. $S \times N / D$ is ergodic if and only if $S \times N / D[N, N]$ is ergodic.

Proof. We can write $L^{2}(S \times N / D)=H \oplus L^{2}(S \times N / D[N, N])$ where $H=\Sigma^{\oplus} L^{2}\left(S ; H_{i}\right)$, and $H_{i}$ is the subspace of $L^{2}(N / D)$ corresponding to an infinite dimensional irreducible subrepresentation $\pi_{i}$ of $\sigma$. Suppose that $S \times N / D$ is not ergodic, so there is a nonconstant $G$-invariant $f \in L^{2}(S \times N / D)$. If $S \times N / D[N, M]$ is ergodic, then $f \notin L^{2}(S \times N / D[N, N])$ and hence, for some $i$, the orthogonal projection of $f$ onto $L^{2}\left(S ; H_{i}\right)$, say $f^{\prime}$, will be nonzero. Then $f^{\prime}$ is also $G$-invariant, 
and it is straightforward that this implies that $\pi_{i} \circ \alpha$ contains the identity cocycle. By Theorem 3.2, this is impossible. Hence $S \times N / D[N, N]$ is not ergodic. The converse assertion is clear.

We remark that conditions under which $S \times N / D[N, N]$ is ergodic are given in [10, Corollary 3.8], as $N / D[N, N]$ is a compact abelian group.

Corollary 3.5. Suppose $\alpha: S \times G \rightarrow N$, and that $S \times N / D$ is ergodic. Then the subspace of $L^{2}(S \times N / D)$ corresponding to the relative discrete spectrum of $S \times N / D$ over $S\left[10\right.$, Definition 5.1] is precisely $L^{2}(S \times N / D[N, N])$. In particular, every finite dimensional subcocycle of $\sigma \circ \alpha$ is one dimensional.

Proof. Let $s \rightarrow V(s)$ be a finite dimensional $G$-invariant subbundle of the product bundle $s \rightarrow L^{2}(N / D)$. If the orthogonal projection of $V(s)$ onto $H_{i}$ is nonzero on a nonnull set, then $\pi_{i} \circ \alpha$ contains a finite dimensional subcocycle representation which contradicts Theorem 3.2. Thus

$$
V(s) \subset L^{2}(N / D[N, N])
$$

for almost all s. Conversely, one can see, as in [10, Example 4.1] that $L^{2}(S \times N / D[N, N])$ has relatively discrete spectrum over $S$.

For completeness, we include the following result.

Proposition 3.6. Suppose $\alpha: S \times G \rightarrow N$ is a cocycle and $S \times N / D$ is ergodic. Then $S \times N / D$ has generalized discrete spectrum over $S[11$, Definition 8.4].

Proof. Let $A_{i}$ be a decreasing sequence of neighborhoods of [e] in $N / D$ with $\cap A_{i}=\{[e]\}$. Then it follows from the fact that $N$ acts distally on $N / D$ [1, Theorem IV.3] (see also the remark in [11, Example 9.7]) that $A_{i}$ is a relative separating sieve for $S \times N / D$ over $S$ [11, Definition 8.5]. The result now follows from [11, Theorem 8.7].

\section{REFERENCES}

1. L. Auslander, L. Green and F. Hahn, Flows on homogeneous spaces, Ann. of Math. Studies, no. 53, Princeton Univ. Press, Princeton, N.J., 1963. MR 29 \#4841.

2. L. Auslander, An exposition of the structure of solvmanifolds, Bull. Amer. Math. Soc. 79 (1973), $227-285$.

3. E. G. Effros, Convergence of closed subsets in a topological space, Proc. Amer. Math. Soc. 16 (1965), 929-931. MR 31 \#6208.

4. The Borel space of von Neumann algebras on a separable Hilbert space, Pacific J. Math 15 (1964), 1153-1164. MR 32 \#2923.

5. Transformation groups and $C^{*}$-algebras, Ann. of Math. (2) 81 (1965), 38-55. MR 30 \#5175.

6. C. Kuratowski, Topologie. Vol. I, 4th ed., Monografie Mat., Tom 20, PWN,Warsaw, 1958. MR 19, 873.

7. G. W. Mackey, Ergodic theory and virtual groups, Math. Ann. 166 (1966), 187207. MR 34 \#1444. 
8. C. C. Moore, Ergodicity of flows on homogeneous spaces, Amer. J. Math 88 (1966), 154-178. MR 33 \#1409.

9. V. S. Varadarajan, Geometry of quantum theory. Vol. II, Van Nostrand, Princeton, N.J., 1970.

10. R. J. Zimmer, Extensions of ergodic group actions, Illinois J. Math. 20 (1976), 373409.

11. Ergodic actions with generalized discrete spectrum, Illinois J. Math. 20 (1976).

DEPARTMENT OF MATHEMATICS, U.S. NAVAL ACADEMY, ANNAPOLIS, MARYLAND 21402 